\title{
Diagnosis of oral cancer by light-induced autofluorescence spectroscopy using double excitation wavelengths
}

\author{
Chih-Yu Wang ${ }^{\mathrm{a}}$, Huihua Kenny Chiang a,*, Chin-Tin Chen ${ }^{\mathrm{b}}$, Chun-Pin Chiang ${ }^{\mathrm{c}}$, \\ Ying-Shiung $\mathrm{Kuo}^{\mathrm{c}}$, Song-Nan Chow ${ }^{\mathrm{b}}$ \\ ${ }^{a}$ Institute of Biomedical Engineering, National Yang-Ming University 155, Section 2, Li-Nung Street, Taipei, Taiwan \\ ${ }^{\mathrm{b}}$ Laser Medicine Research Center, National Taiwan University, Taipei, Taiwan \\ ${ }^{\mathrm{c} S}$ Shool of Dentistry, National Taiwan University, Taipei, Taiwan
}

Received 22 September 1998; accepted 14 November 1998

\begin{abstract}
A cancer diagnostic algorithm, light-induced autofluorescence spectroscopy using double excitation wavelengths, was employed for distinguishing between cancerous and normal oral mucosa. For emission spectra at the shorter excitation wavelengths (280, 290, and $300 \mathrm{~nm}$ ), the ratio between the area under $325-335 \mathrm{~nm}$ and the area under $465-475 \mathrm{~nm}$ was calculated. In the same way, for emission spectra at the longer excitation wavelengths $(320,330$, and $340 \mathrm{~nm})$, the ratio between the area under $375-385 \mathrm{~nm}$ and the area under 465-475 nm was calculated. Receiver operating characteristic curves were used to evaluate the performance of algorithms using single and the double (by combining shorter and longer) excitation wavelengths. The results showed that better performance, up to sensitivity $81.25 \%$, specificity $93.75 \%$, and positive predictive value $92.86 \%$, could be achieved by using the double excitation wavelengths. The present study can be useful as a basis for further investigation on in vivo autofluorescence measurement and analysis using double excitation wavelength. (C) 1999 Elsevier Science Ltd. All rights reserved.
\end{abstract}

Keywords: Autofluorescence spectroscopy; Double excitation wavelengths; Oral cancer

\section{Introduction}

Oral cancer has become one of the leading malignancies in many Asian countries [1], where harmful habits such as smoking, heavy alcohol consumption, and betel quid (BQ) chewing have been identified as possible causes for the increased incidence $[2,3]$. Oral premalignant lesions (such as leukoplakia and eryplakia) and malignant lesions (such as squamous cell carcinoma) are frequently found among BQ chewers, giving urgency to the need to develop a screening and diagnostic technique for early-stage oral cancer.

Among the diagnostic techniques for identifying oral premalignant and malignant lesions, pathological examination of incisional biopsy specimen is the most reliable. Even experienced clinicians, however, cannot easily select a representative site for biopsy. Therefore, a technique for non-invasively detecting cancer or helping

\footnotetext{
* Corresponding author. Tel.: +886-2-28267027; fax: +886-228210847; e-mail: chiang@bme.ym.edu.tw
}

the clinician choose the appropriate site for biopsy can save patients from multiple biopsies and allow a broader range of diagnoses, which can aid early detection of oral cancer.

In contrast to conventional biopsy techniques, lightinduced autofluorescence spectroscopy can be conducted to characterize tissues or to detect cancers without removing them, and the analysis results can be obtained in near real-time. Studies have shown that autofluorescence spectroscopy is useful for distinguishing cancerous from normal tissues in a variety of organ systems, including the human lung and breast [4,5], bronchus [6], colon $[7,8]$, cervix $[9,10]$, esophagus [11] and head and neck $[12,13]$. Some typical fluorophores in human tissues, such as proteins and amino acids (including tryptophan, tyrosin, phenylalanine, and elastin), purines, pyrimidines, and nucleic acids (including adenine, adenosine, guanine, guanosine, DNA, and RNA) absorb ultraviolet light near $280 \mathrm{~nm}$ and have autofluorescence peaks near 340, 360, and $390 \mathrm{~nm}$ $[14,15]$. This finding suggests that shorter excitation wavelengths, such as 280, 290, and $300 \mathrm{~nm}$, could be 
efficient excitation wavelengths for spectroscopic research involving human tissues.

On the other hand, significant differences between the autofluorescence spectra of normal and cancerous tissues have been found near $340 \mathrm{~nm}$ excitation wavelength. Schomacker et al. [16] and Ramanujam et al. [17] have shown that the decrease in collagen and the increase in NADH and hemoglobin in cancer tissue have significant effects on tissue autofluorescence changes when a $337-\mathrm{nm}$ nitrogen laser is used as the excitation light source. This finding suggests that longer excitation wavelengths, such as 320,330 , and $340 \mathrm{~nm}$, might be other useful excitation wavelengths. Thus, we adopted a combination of a shorter excitation wavelength group of $280,290,300 \mathrm{~nm}$, and a longer excitation wavelength group of 320,330 , and $340 \mathrm{~nm}$ to evaluate the feasibility of the double excitation wavelength algorithm in this study.

A single excitation wavelength has been widely used in autofluorescence studies [4-13,17], but the information obtained from the emission spectra is limited if only one excitation wavelength is employed. In a work about spectroscopic diagnosis of cervical intra-epithelial neoplasia (CIN) in vivo, Ramanujam et al. [18] have shown that fluorescence spectra at multiple excitation wavelengths are essential for the detection and differential diagnosis of squamous intra-epithelial lesions (SILs) at colposcopy. Because human tissues contain several kinds of fluorophores and each fluorophore has its own excitation-emission characteristics, a single excitation wavelength may not be sufficient to reflect the fluorescence structure in a complicated human tissue sample. Multiple excitation wavelengths could be useful for addressing the above mentioned limitation. For this purpose, light-induced autofluorescence spectroscopy using double excitation wavelengths was proposed for identifying oral cancer, and its performance as a diagnostic tool was compared to that of an algorithm using a single excitation wavelength.

\section{Materials and methods}

\subsection{Sample source and processing}

A total of 32 oral tissue samples from 16 patients (one cancerous and one normal oral mucosal tissue sample from each patient) undergoing oncological surgery at Taiwan University Hospital, Taiwan, were used in this study. Informed consent of the subjects and institutional review board approval were obtained before the experiment. Immediately after being resected, all samples were snap frozen in cryomatrix (Shandon Scientific Ltd., UK), stored initially in liquid nitrogen, and then later at $-70^{\circ} \mathrm{C}$ in an ultralow temperature freezer until spectroscopic measurement was performed. Prior to measurement, the frozen samples were taken out of the freezer, cut into pieces 4-6 $\mathrm{mm}$ in diameter, brought to room temperature, and rinsed with $4^{\circ} \mathrm{C}$ phosphatebuffered saline (PBS) to remove the remaining cryomatrix. The tissue samples were then mounted on a quartz slide for spectroscopic measurement. After autofluorescence measurements all tissue samples were submitted to be fixed, embedded, sectioned, and hematocylin and eosin (H\&E) stained. The section slides were reviewed by oral pathologists, and all the cancerous tissues were determined by histological examination to be squamous cell carcinoma. These results were then used as the standard for checking the accuracy of the spectroscopic classification.

\subsection{Autofluorescence spectroscopy}

Autofluorescence spectra were measured with a standard luminescence spectrometer (SLM Instruments, Urbana, IL, USA). The excitation light source, a monochromator with a 150 -W ozone-free Xenon lamp, was incident directly to the surface of samples, and the emission spectra were collected at a $37^{\circ}$ angle with respect to the excitation beam. Such geometry can minimize the collection of reflected excitation light for semi-opaque materials or solid tissues [19]. The excitation light was focused as a spot, $4 \mathrm{~mm}$ long and $1 \mathrm{~mm}$ wide, on the center of the quartz slide. The emission spectra were measured from $20 \mathrm{~nm}$ above the excitation wavelength to $20 \mathrm{~nm}$ below twice the excitation wavelength to avoid the collection of the first and second harmonics of the excitation light.

\subsection{Test indices}

The performance of each algorithm in this study was evaluated in terms of three test efficiency indices: specificity, sensitivity, and the positive predictive value. The tested samples were assumed to belong to either normal population $N$ or cancerous population $C$. The test results were dichotomized as positive $(y=1$, which represents cancerous) or negative $(y=0$, which represents normal) by selecting an appropriate cutoff point. According to Albert et al. [20], specificity is the probability of normal tissues whose diagnostic results are negative, sensitivity is the probability of cancerous tissues whose diagnostic results are positive, and the positive predictive value is the probability that the tissues are cancerous when the tested outcome is positive. Their mathematical representations are as follows:

specificity $=\operatorname{Pr}(y=0 \mid N)$,

sensitivity $=\operatorname{Pr}(y=1 \mid C)$, and

positive predictive value $=\operatorname{Pr}(C \mid y=1)$, 
where $\operatorname{Pr}(\cdot \mid \cdot)$ denotes the conditional probability. These test indices are widely used in differential diagnosis for clinical applications [20].

\subsection{Canonical discriminant function for a two-variable discrimination problem}

For a two populations discrimination problem with two independent variables (denoted by $X_{1}$ and $X_{2}$ ), the simplest approach for getting a discriminant function $Z$ is to take a linear combination of the two variables:

$Z=a_{1} X_{1}+a_{2} X_{2}$.

The canonical discriminant function, a way to optimally discriminate the two populations, is obtained by choosing the coefficients $a_{1}$ and $a_{2}$ to maximize the $F$ ratio of the one-way analysis of variance for the variation within and between the two populations related to variable $Z$ [21]. The canonical discriminant function was used in the double excitation wavelength algorithm to determine a decision line for separating the normal and cancerous tissues in this study.

\subsection{Receiver operating characteristic (ROC) curves}

The ROC curve is a graphic way that relates specificity and sensitivity to different thresholds that separate two populations. The ROC curve is obtained by plotting the false positive rate (1-specificity) versus the sensitivity for various values of thresholds [20]. In general, an effective diagnostic algorithm has a ROC curve located near the upper left corner of the plot, at which both the specificity and sensitivity are high. The ROC curve could thus be used to compare and evaluate the performances of different algorithms. The curve is also helpful for determining the threshold if the desired specificity and sensitivity are selected.

\section{Results}

\subsection{Preliminary studies}

A control experiment showed that the autofluorescence intensities of PBS and cryomatrix were at least 1000 times less than that of the oral tissues at all wavelengths in this investigation. For each sample, a less than $5 \%$ change in autofluorescence intensity was observed between two successively measured spectra, indicating a non-significant photobleaching effect.

\subsection{Fluorescence spectra}

The intensities of the spectrum at each wavelength were divided by the integrated area under the total spectrum for normalization purposes. Panels in Fig. 1 depict the typical normalized emission spectra of both normal and cancerous oral tissues at 280-, 290-, 300-, 320-, 330-, and 340-nm excitation wavelengths, respectively. As shown in Fig. 1a-c, two emission peaks appeared at 330 and $470 \mathrm{~nm}$ at 280-, 290-, and 300-nm excitations (the shorter wavelength set), whereas in Fig. 1d-f two prominent peaks appeared at 380 and 460 $\mathrm{nm}$ at 320-, 330-, and 340-nm excitations (the longer wavelength set). For each excitation, the ratios between the integrated area of $\pm 5 \mathrm{~nm}$ around two peaks were calculated for the spectrum of each sample. These ratio values were employed for discriminating between normal and cancerous tissues.

\subsection{Comparison between performances of double and single excitation wavelength using ROC curves}

For the autofluorescence spectrum at 280-, 290-, and 300-nm excitation wavelengths, the ratios between the areas under $330 \pm 5$ and $470 \pm 5 \mathrm{~nm}$ (denoted as $A_{330 \pm 5} /$ $\left.A_{470 \pm 5}\right)$ were calculated. Also for the autofluorescence spectrum at 320-, 330-, and 340-nm excitation wavelengths, the ratios between the areas under $380 \pm 5$ and $460 \pm 5 \mathrm{~nm}$ (denoted as $A_{380 \pm 5} / A_{460 \pm 5}$ ) were calculated. The ROC curves for the single excitation wavelength algorithm were drawn by successively changing the thresholds for discriminating normal and cancerous tissues. On the other hand, the double excitation wavelength algorithm was formed by pairing 280-, 290-, and 300-nm (the shorter wavelength set) with 320-, 330and 340-nm excitations (the longer wavelength set). The slope of the decision line (i.e. the threshold) was determined by using the canonical discriminant function [21], and the ROC curve was drawn by continuously shifting the decision line in parallel. Panels in Fig. 2 show the ROC curves with the use of single and double excitation wavelength algorithms. A comparative evaluation of the ROC curves in these nine panels indicated that for an appropriate sensitivity (e.g. $80 \sim 85 \%$ ), the double excitation wavelength algorithm had a lower false-positive rate (i.e. higher specificity) than did the single excitation wavelength algorithm. Therefore, we could conclude that the double excitation wavelength algorithm might perform better than the single excitation wavelength algorithm for oral cancer detection, as observed in this study.

Fig. 3a and $\mathrm{b}$ depict the results of the single excitation wavelength algorithm with 280- and 330-nm excitation, respectively; the discriminant lines were drawn according to the selected thresholds shown in Fig. 2b. It is noticeable that with the double excitation wavelength algorithm (Fig. 3c) the oral cancer samples are generally located at the upper left corner, whereas the normal oral mucosa samples are located at the lower right corner. The threshold selected for the desired sensitivity and 


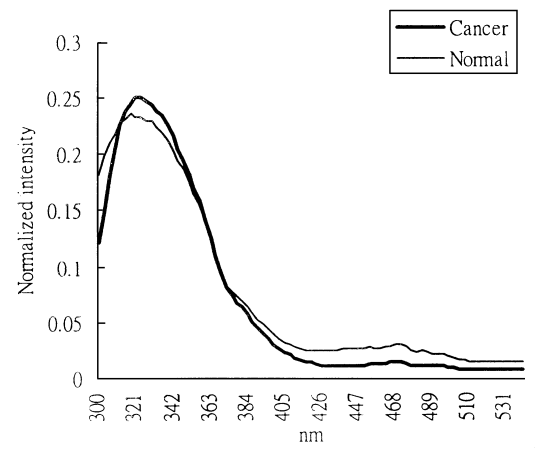

(a)

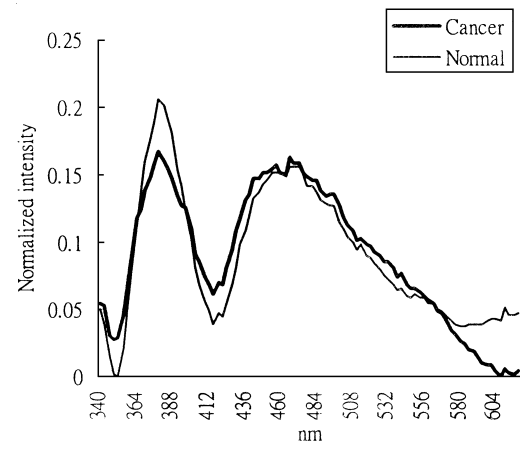

(d)

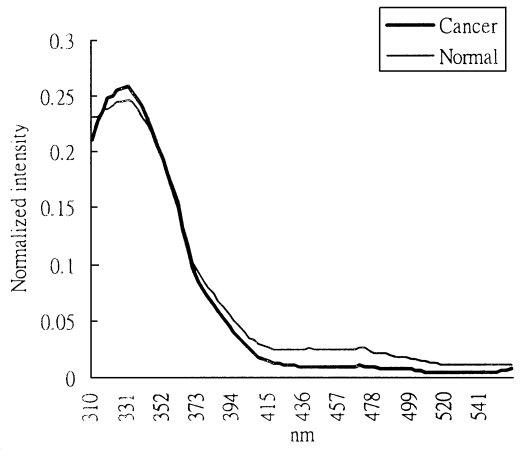

(b)

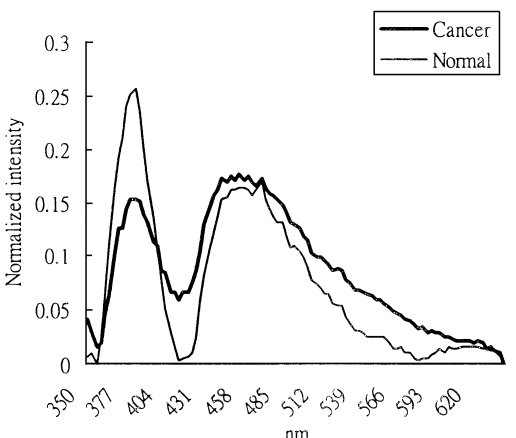

(e)

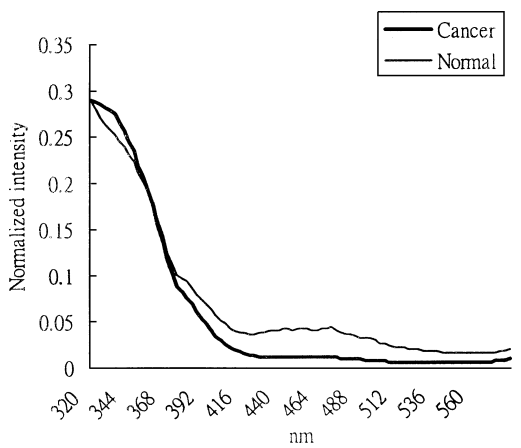

(c)

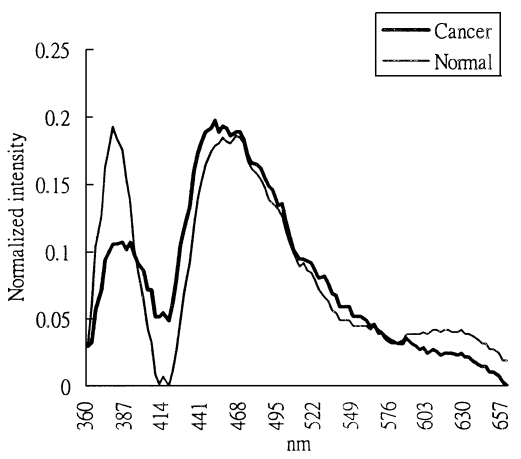

(f)

Fig. 1. Typical normalized fluorescence spectra for oral tissues at (a) 280-nm, (b) 290-nm, (c) 300-nm, (d) 320-nm, (e) 330-nm, and (f) 340-nm excitations.

specificity is a straight line that separates these samples into two groups. In general, the double excitation wavelength algorithm performed better than the single excitation wavelength algorithm, as illustrated by the improvement in the specificity and positive predictive value, for the same sensitivity (Table 1).

\section{Discussion}

The results of this study showed that with appropriate sensitivity the double excitation wavelength algorithm had higher specificity than did the single excitation wavelength algorithm, suggesting that the use of this algorithm might decrease the false positive rate and consequently help patients avoid unnecessary biopsy or treatment. Furthermore, it could not only broaden the scale of cancer diagnoses but also help less experienced practitioners choose representative locations for histological analysis. Thus, with further development, the double excitation wavelength algorithm could be useful for clinical implementation.

The major factors that contribute to autofluorescence changes in human neoplasia remain controversial. For instance, in a similar intrinsic fluorescence study on colonic cancer tissues, Yakshe et al. [22] have contended that changes in autofluorescence spectra between normal and cancerous colonic tissues probably arise from rearrangement of tissue architecture. However, in a study on colonic adenoma, Cothren et al. [23] suggested that architectural differences between flat and polypoid mucosa are not the only factors contributing to autofluorescence changes. Recently, in a study based on the laser-induced fluorescence microscopy of normal tissues and adenomas of colon, Romer et al. [8] argued that the autofluorescence differences are not attributable to the gross architecture alone, but also to the intrinsic changes in fluorophores of colonic adenomas. These studies indicate that the mechanisms causing changes between normal and abnormal tissue autofluorescence are quite complex, requiring further study of the fluorescent structure for their understanding. Important information pertaining to fluorescent structure might be lost with the use of a single excitation wavelength, but might be obtained with other excitation wavelengths [24], and thus enhanced the diagnostic content of tissue spectra at single excitation wavelength [17]. For this reason, double or multiple excitation wavelengths should aid in exploring these issues.

The favorable discriminant results obtained by employing the double excitation wavelength algorithm might be explained as follows. First, human tissues comprise various fluorophores, and each fluorophore has a different autofluorescence spectra pattern at 


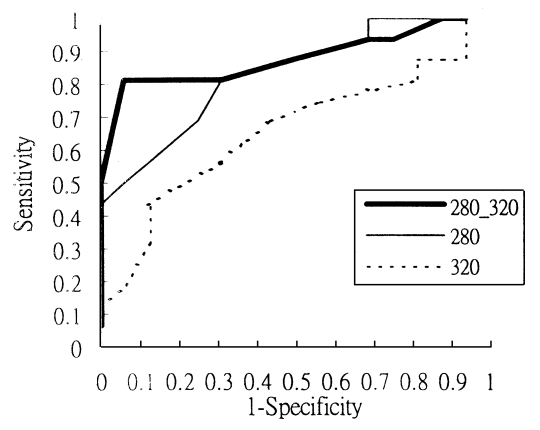

(a)

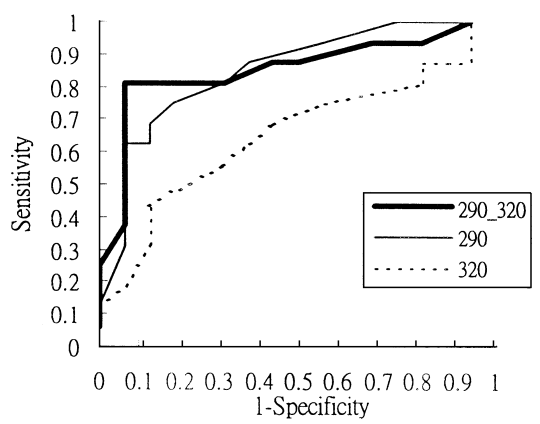

(d)

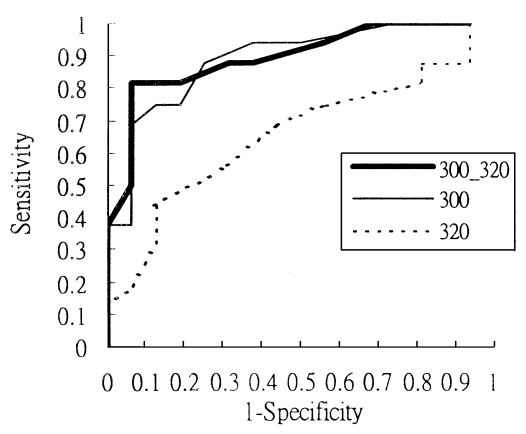

(g)

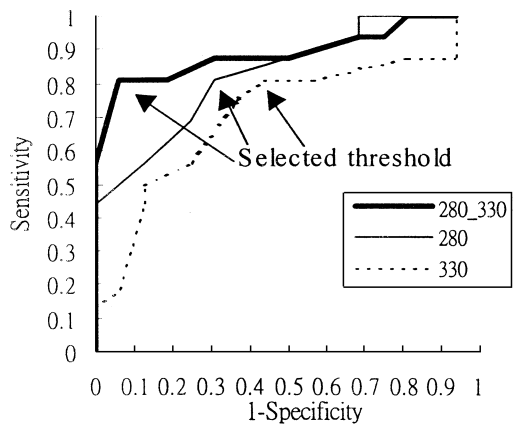

(b)

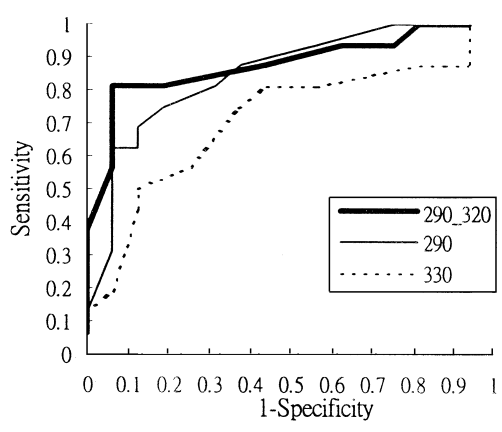

(e)

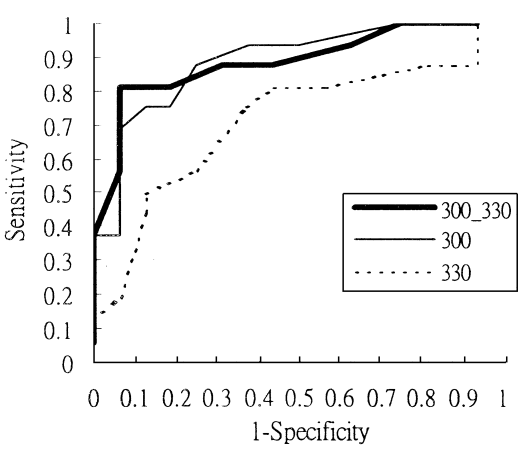

(h)

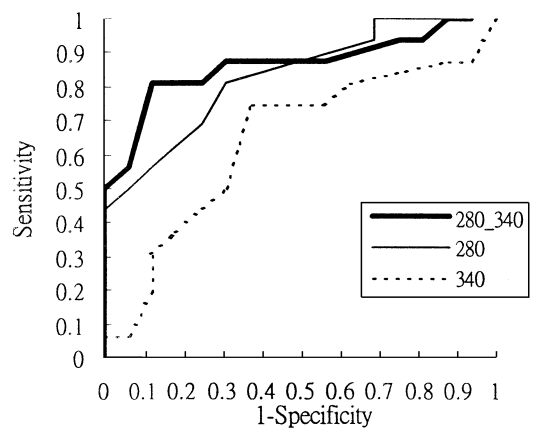

(c)

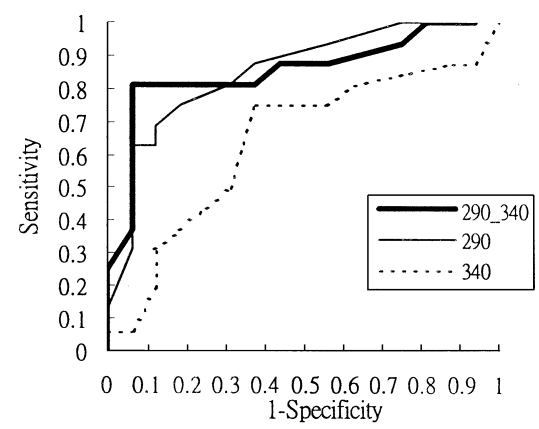

(f)

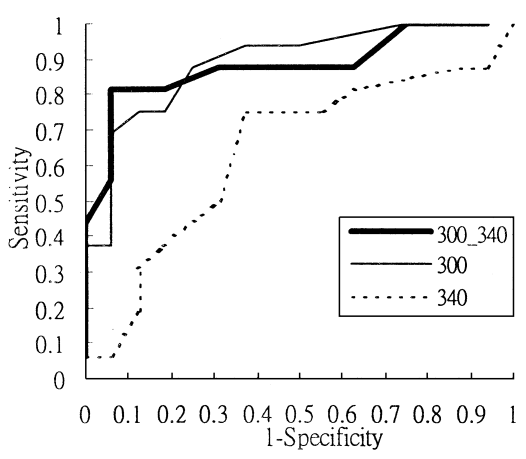

(i)

Fig. 2. The receiver operating characteristic (ROC) curves of double wavelength excitation algorithm (combining 280-, 290-, 300-nm and 320-, 330-, 340-nm excitations).

different excitations. The double excitation wavelength, due to the combined characteristics of two different spectra, could provide more diagnostic information. Second, two variables were used in the double excitation wavelength algorithm. The first variable, the value $A_{330 \pm 5} / A_{470 \pm 5}$ of emission spectra at 280-, 290-, and $300-\mathrm{nm}$ excitations, represented the ratio between the concentration of some amino acids and NADH. The second variable, the value $A_{380 \pm 5} / A_{460 \pm 5}$ of emission spectra at 320-, 330-, and 340-nm excitations, represented the ratio between the concentration of collagen and NADH. The combination of the two variables applied additional information to the discrimination. Furthermore, the use of two variables could increase the degree of freedom, and thus better performance could be obtained than with the single excitation wavelength algorithm.

The effectiveness of autofluorescence spectroscopy diagnostic techniques greatly depends on the selection of the excitation wavelengths. For autofluorescence spectroscopy, ultraviolet lasers have some excellent properties, such as narrow bandwidth, high intensity, small spot size, and short pulse operation. However, because the number of appropriate ultraviolet lasers is limited, the selection of excitation wavelengths is restricted as well. Therefore, a wavelength-tunable light source might be more appropriate for an autofluorescence spectroscopy study. An autofluorescence 


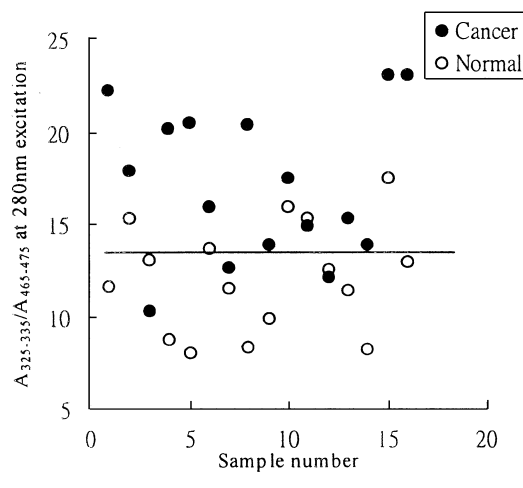

(a)

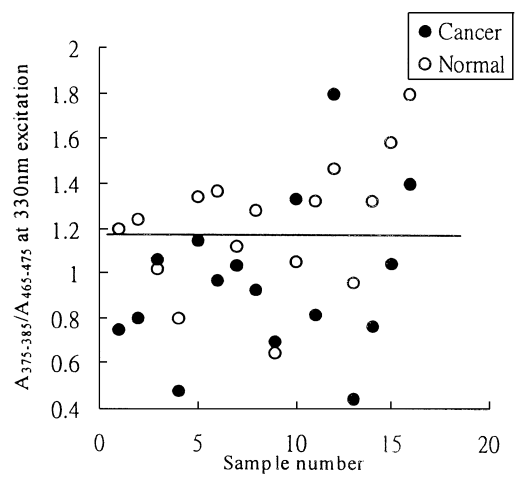

(b)

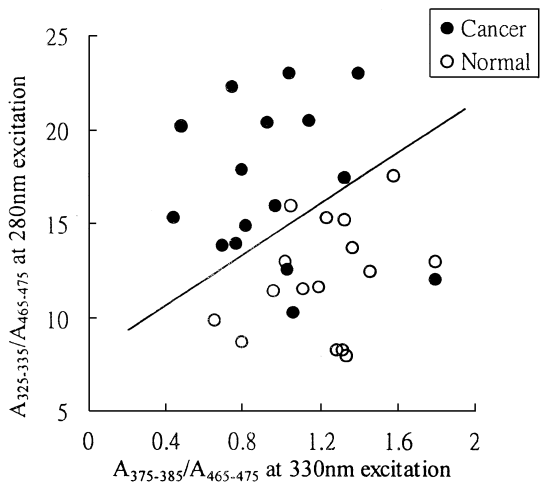

(c)

Fig. 3. The scatter plots of the discrimination results for normal and cancerous tissues by individual and pairing $280-$ with 330 -nm excitations at selected thresholds.

Table 1

The sensitivity, specificity, and positive predictive value of 'single wavelength excitation' and 'double-wavelength excitation' algorithms at selected thresholds, respectively

\begin{tabular}{lccc}
\hline $\begin{array}{l}\text { Excitation } \\
\text { wavelength }(\mathrm{nm})\end{array}$ & $\begin{array}{c}\text { Sensitivity } \\
(\%)\end{array}$ & $\begin{array}{c}\text { Specificity } \\
(\%)\end{array}$ & $\begin{array}{c}\text { Positive predictive } \\
\text { value }(\%)\end{array}$ \\
\hline 280 & 81.25 & 68.75 & 72.22 \\
330 & 81.25 & 62.50 & 68.42 \\
280 (pairs) 330 & 81.25 & 93.75 & 92.86 \\
\hline
\end{tabular}

measurement system consisting of a Xenon lamp and a monochrometer-based excitation light source, an intensified charge coupled device (ICCD) camera, and a Y-type UV fiber could be very useful for clinical oral cancer diagnosis in the future as double or multiple excitation wavelength algorithms are developed.

The present study adopted a simple index, the ratios between the integrated area of $\pm 5 \mathrm{~nm}$ around two peaks for the spectrum of each sample, for discriminating normal and cancerous tissues at multiple excitation wavelengths. It was an uncomplicated and reasonable approach for monitoring some kinds of main fluorophores alterations (or their mixed effect) in cancer tissues related to these emission peaks, but might have the drawback for lacking consideration of other important chemical materials that were not related to these peaks of autofluorescence spectra. Another limit of the current-used method was that the choice of peaks and range for ratio was not easy to determine. To prevent these problems, some researchers employed multivariate statistical algorithm to extract clinically important information from tissue spectra $[25,26]$. This approach is more complicated in calculation, but can provide more information of autofluorescence spectra of tissues. The analysis of autofluorescence spectra at multiple excitation wavelengths with multivariate statistical algorithm is worthy of study in future investigation.
This study did not consider environmental conditions, such as blood infiltration, saliva secretion, and oral cavity temperature, and thus differences between an in vivo and an ex vivo study are possible. Different spectra might be obtained when in vivo and ex vivo experiments are conducted [27], and this difference might have some effect on the classification of normal and cancerous oral tissues. Therefore, an in vivo study on this topic is needed to evaluate the clinical applicability of the double excitation wavelength algorithm. Still, this ex vivo experiment can be useful as a pre-study, a basis for clinical experiments and further investigation.

\section{Uncited reference:}

[16]

\section{References}

[1] Department of Health, the Executive Yuan, ROC. Cancer Registry Annual Report, Republic of China, 1996.

[2] Tennekoon GE, Bartlett GC. Effect of betel chewing on the oral mucosa. The British Journal of Cancer 1969;23:39-43.

[3] Ko YC, Huang YL, Lee CH, et al. Betel quid chewing, cigarette smoking and alcohol consumption related to oral cancer in Taiwan. Journal of Oral Pathology and Medicine 1995;24:450-3.

[4] Alfano RR, Tang GC, Pradhan A, Lam W, Choy DSC, Opher A. Fluorescence spectra from cancer and normal human breast and lung tissues. IEEE Journal of Quantum Electronics 1987;QE23(10):1806-11.

[5] Tang GC, Pradhan A, Alfano RR. Spectroscopic differences between human cancer and normal lung and breast tissues. Lasers in Surgery and Medicine 1989;9:290-5.

[6] Hung J, Lam S, LeRiche JC, Palcic B. Autofluorescence of normal and malignant bronchial tissue. Lasers in Surgery and Medicine 1991;11:99-105.

[7] Bottiroli G, Corce AC, Locatelli D, Marchesini R, Pignoli E, Tomatis S, Cuzzoni C, Palma SD, Dalfante M, Spinelli P. Natural fluorescence of normal and neoplastic human colon: a comprehensive 'ex vivo' study. Lasers in Surgery and Medicine 1995; 16:48-60. 
[8] Romer TJ, Fitzmaurice M, Cothren RM, Richards-Kortum R, Petras R, Sivak MV, Kramer LR, Romer TJ. Laser-induced fluorescence microscopy of normal colon and dysplasia in colonic adenomas: implication for spectroscopic diagnosis. The American Journal of Gastroenterology 1995;90(1):81-7.

[9] Mahadevan A, Mitchell MF, Silva E, Thomsen S, Richard-Kortum RR. A study of the fluorescence properties of normal and neoplastic human cervical tissue. Lasers in Surgery and Medicine 1993;13:647-55.

[10] Ramanujam N, Mitchell MF, Mahadevan A, Thomsen S, Silva E, Richards-Kortum RR. Fluorescence spectroscopy: a diagnostic tool for cervical intraepithelial neoplasia (CIN). Gynecologic Oncology 1994;52:31-8.

[11] Vo-Dinh T, Panjehpour M, Overholt BF, Farris C, Bukley III FP, Sneed R. In vivo cancer diagnosis of the esophagus using differential normalized fluorescence (DNF) indices. Lasers in Surgery and Medicine 1995;16:41-7.

[12] Kolli VR, Shaha AR, Savage HE, Sacks PG, Casale MA, Schantz SP. Native cellular fluorescence can identify changes in epithelial thickness in-vivo in the upper aerodigestive tract. American Journal of Surgery 1995;170:495-8.

[13] Kolli VR, Savage HE, Yao TJ, Schantz SP. Native cellular fluorescence of neoplastic upper aerodigestive mucosa. Archives of Otolaryngology — Head \& Neck Surgery 1995;121:1287-92.

[14] Alfano RR, Das BB, Cleary J, Prudent R, Celmer EJ. Light sheds light on cancer-distinguishing malignant tumours. Bulletin of the New York Academy of Medicine 1991;67:143-50.

[15] Sliberberg MB, Savage HE, Tang GC, Sacks PG, Alfano RR, Schantz SP. Detecting retinoic acid-induced biochemical alteration in squamous cell carcinoma using intrinsic fluorescence spectroscopy. Laryngoscope 1994;104:278-82.

[16] Schomaker KT, Frisoli JK, Compton CC, Flotte TJ, Richter JM, Nishioka S, Deutsch TF. Ultraviolet laser-induced fluorescence of colonic tissue: basic biology and diagnostic potential. Lasers in Surgery and Medicine 1992;12:63-78.

[17] Ramanujam N, Mitchell MF, Mahadevan A, Thomsen S, Maloica A, Wright T, Atkinson N, Richards-Kortum R. Spectroscopic diagnosis of cervical intraepithelial neoplasia (CIN) in vivo using laser-induced fluorescence spectra at multiple excitation wavelengths. Lasers in Surgery and Medicine 1996;19:63-74.

[18] Ramanujam N, Mitchell MF, Mahadeven A, Warren S, Thomsen S, Silva E, Richards-Kortum R. In vivo diagnosis of cervical intraepithelial neoplasia using 337-nm-excitation laser-induced fluorescence. Proceedings of the National Academy of Sciences, USA 1994;19:10 193-7.

[19] Willard HH, Merritt LL, Dean JA, Selttle FA. Instrumental Methods of Analysis, p. 112-113. USA: Litton Educational Publishing, 1981.

[20] Albert A, Harris EK. Multivariate Interpretation of Clinical Laboratory Data, p. 76-82. USA: Marcel Dekker, 1987.

[21] Manly BFJ. Multivariate Statistical Methods: a Primer, Chapter 8, 2nd Edition. London: Chapman and Hall, 1994.

[22] Yakshe PN, Bonner RF, Patterson R. Laser-induced fluorescence spectroscopy (LIFS): can it be used in the diagnosis and treatment of colonic malignancies? The American Journal of Gastroenterology 1989;84:1199 (abstract).

[23] Cothren RM, Richards-Kortum RR, Sivak MV, et al. Gastrointestinal tissue diagnosis by laser induced fluorescence spectroscopy at endoscopy. Gastrointestinal Endoscopy 1990;36: $105-11$.

[24] Ramanujam N, Mitchell MF, Mahadevan-Jansen A, Thomsen SL, Staerkel G, Malpica A. Cervical precancer detection using a multivariate statistical algorithm based on laser-induced fluorescence spectra at multiple excitation wavelengths. Photochemistry and Photobiology 1996;64(4):720-35.

[25] Wang CY, Chen CT, Chiang CP, Young ST, Chow SN, Chiang HK. Partial least-squares discriminant analysis on autofluorescence spectra of oral carcinogenesis. Applied Spectroscopy 1998;52(9): 1190-6.

[26] Wang CY, Chen CT, Chiang CP, Young ST, Chow SN, Chiang HK. A probability-based multivariate statistical algorithm for the autofluorescence spectroscopic identification of oral carcinogenesis. Photochemistry and Photobiology (submitted).

[27] Mahadevan A. Fluorescence and Raman spectroscopy for diagnosis of cervical precancers. Ph.D. thesis, The University of Texas at Austin, 1996. 